\title{
A Controversial Issue: The Intelligence of Matter as Residue? A Possible Understanding
}

\author{
Gianfranco Minati* \\ Italian Systems Society, Italy \\ *Corresponding author: Gianfranco Minati, Italian Systems Society, 20161 Milan, Italy
}

\section{ARTICLE INFO}

Received: 杫 November 13, 2019

Published: ${ }^{\Perp}$ November 20, 2019

Citation: Gianfranco Minati. A Controversial Issue: The Intelligence of Matter as Residue? A Possible Understanding. Biomed J Sci \& Tech Res 23(1)-2019. BJSTR. MS.ID.003848.

\section{ABSTRACT}

In this short paper we consider the insufficiency of Darwin's evolutionism to deal with unanswered questions considered by neo Darwinism, such as genetic mutations. From this perspective, we consider the possibility of a sort of intelligence of matter as a property rather than the product of external, invasive, divine interventions. Through the Big Bang's irreversible door, enormous energy passed, as stated by Lemaitre, and also, we conjecture, the passing of properties. This is the case for the embedded properties of matter, such as entanglement and, in the quantum vacuum, the emergence of collective long-range excitations named Nambu-Goldstone bosons (NGBs), and intelligence. This concept could open new philosophical and scientific research avenues on property convey ability without explicit mediatory processes. An example of this phenomenon is non-intermediated remote synchronization and variable long-range correlations.

\section{Introduction}

\section{The Big Bang: The Door Closed Behind Us}

The Big Bang Theory, introduced in 1927 by the Belgian Catholic priest Georges Lemaître and proposed as a model for the expanding universe, is currently the leading explanation about how the universe began [1]. Shortly, the theory says that the universe started with a small singularity and then inflated over the next 13.8 billion years to become the cosmos. Lemaître says "if we go back in the course of time, we must find fewer and fewer quanta, until we find all the energy of the universe packed in a few or even in a unique quantum". This is not an article on theoretical physics but, instead, an article on the philosophy of matter inspired by different views, such as the Big Bang Theory, and approaches like constructivism, creativism, evolutionism, and objectivism.

\section{The Intelligence of Matter}

In particular, we move from the fact that single approaches such as the four listed above are not considerable exhaustive but, rather, both as partial approaches and approaches to be contextually used to achieve effectiveness. We mention how the subject of matter relates to a large variety of aspects, such as antimatter, biological matter, dark matter, inert matter, living and non-living matter, matter-energy, mind and matter, and so on. We mention how approaches based on considering material entities and particles as fields (for instance, fields of forces) have a long tradition in physics, from Faraday and Maxwell to general relativity. Within this conceptual framework, particles are considered as regions of space where a field has a particularly high intensity.

In QFT, the quantum vacuum is an entity that precedes matter, so it also must precede space and time [2,3]. In this way, the classic idea of matter as a substratum, a metaphysical entity allowing physical existence and possessing properties, loses consistency. As intelligence of matter (classic matter, fields, quantum matter) we consider its emergent properties and its behavior. For instance, chemical reactions, phase transitions, and the ability to acquire emergent properties in conditions of theoretical incompleteness [4] such as coherence, long-range correlation, self-similarity, synchronization, power laws, and life [5]. We will limit ourselves here to considering living matter, even if our ideas could be generically and tentatively extended to non-living matter. Furthermore, living matter must be dissipative where its stability may be required -in short, living matter is reached and maintained by a system far from equilibrium through a continuous process of resilience and balancing (e.g., autocatalytic reactions and processes of self-repairing, self-reproducing, and self-regenerating). 


\section{Evolution and Creationism}

At this point, we define intelligence of matter by its ability to invent (make emergent?), (actuate itself?) acquire, select, optimize, and reuse options initiating subsequent evolutionary (unlimited?) processes. Here, we refer to problems of genetic mutations and neo Darwinism. Does evolution occur after intelligent, fundamental choices? This subject is considered by academic books [6] and journal articles, such as Haqq-Misra [7]. We already considered the magnificence of the incompleteness of Nature allowing emergence [4], such as the theoretical principles of uncertainty and indeterminacy in Physics, the simultaneous occurrence of quantum and non-quantum phenomena, the Dynamic Usage of Models (DYSAM)[8], and the multiplicity of phenomena, such as biological and psychological, algebraical and geometrical, military, economical, and political. In this conceptual context we consider the need for combined, contextual usages of opposite approaches, such as constructivism and objectivism and, in our case here, creationism and evolutionism. The intelligence of evolutionary processes has been considered in various ways, such as learning optimizing energetic process. However, where does this creative intelligence come from, apart from considering invasive interventions that are inevitably external and scientifically difficult to accept?

\section{Intelligence as Residue?}

Can the intelligence of matter be thought to have existed since the origin of matter? Is all energy of the universe, as mentioned by Lemaitre, concentrated in a single quantum point, coalescing with properties such as intelligence? The door of the singularity of the Big Bang is closed behind us. However, we may consider not only energetic phenomena but, possibly, the unidirectional movement away from the door of an unavoidable space of properties, such as quantum entanglement and intelligence, since "... in the quantum vacuum, each perturbation causes the emergence of collective longrange excitations named Nambu-Goldstone bosons (NGBs) which coordinate the behavior of individual components of the system, so as to keep general coherence" [3].

\section{ISSN: 2574-1241}

DOI: $10.26717 /$ BJSTR.2019.23.003848

Gianfranco Minati. Biomed J Sci \& Tech Res

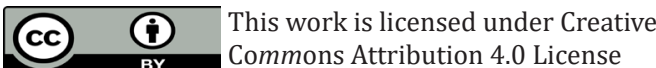

Submission Link: https://biomedres.us/submit-manuscript.php
Are we materialized intelligence recognizing its origins and itself? This could open philosophical and scientific research avenues on property convey ability without explicit mediatory process. An example of this phenomenon is the non-intermediated remote synchronization [9], variable long-range correlations, and the ultra-high dilution in physiology and physics [10].

\section{Acknowledgement}

None.

\section{Conflict of Interest}

No conflict of interest.

\section{References}

1. Lemaitre G (1931) The Beginning of the World from the Point of View of Quantum Theory. Nature 127: 706.

2. Preparata G (2002) Introduction to a realistic quantum physics. Singapore: World Scientific, Singapore.

3. Minati G, Pessa E (2018) From Collective Beings to Quasi-Systems. Springer: New York, USA.

4. Minati G (2016) Knowledge to Manage the Knowledge Society: The Concept of Theoretical Incompleteness, Systems 4(3): 1-19.

5. Minati G (2019) Phenomenological structural dynamics of emergence: an overview of how emergence emerges. In the Systemic Turn in Human and Natural Sciences. A Rock in The Pond, Urbani Ulivi L., Ed., Springer: New York, USA, pp. 1-39.

6. Meyer SC (2014) Darwin's Doubt: The Explosive Origin of Animal Life and the Case for Intelligent Design. Harper One.

7. Haqq Misra J (2016) A better theory of intelligent design, The Boston Globe.

8. Minati, G, Pessa E (2006) Collective Beings. Springer: New York, NY, USA.

9. Minati L (2015) Remote synchronization of amplitudes across an experimental ring of non-linear oscillators. Chaos 25(12): 123107123112 .

10. Endler PC (2010) (Ed.). Ultra High Dilution: Physiology and Physics. Kluwer Academic Publishers, Dordrecht, The Netherlands.

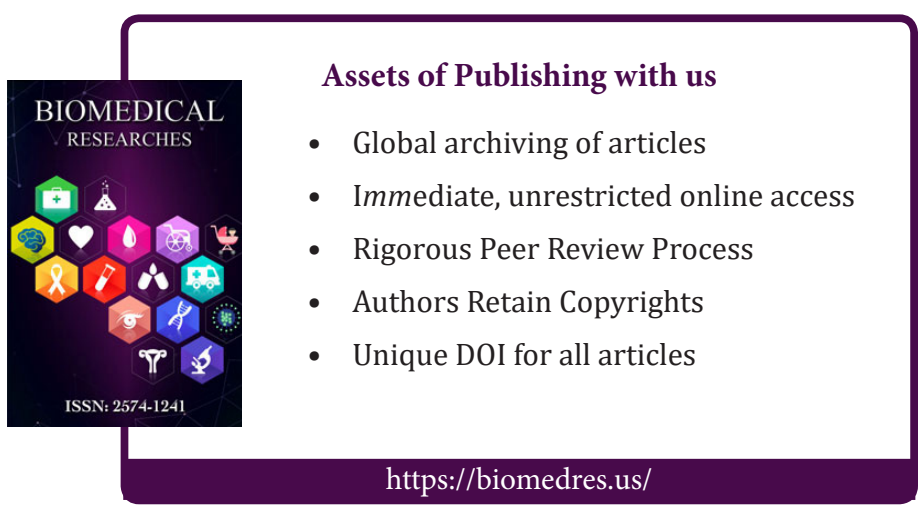

